\title{
The Parabolic Infinite-Laplace Equation in Carnot Groups
}

\section{Thomas Bieske \& Erin Martin}

\begin{abstract}
By employing a Carnot parabolic maximum principle we show the existence and uniqueness of viscosity solutions to a class of equations modeled on the parabolic infinite Laplace equation in Carnot groups. We show the stability of solutions within the class and examine the limit as $t$ goes to infinity.
\end{abstract}

\section{Motivation}

In Carnot groups, the following theorem has been established.

Theorem $1.1[3 ; 14 ; 5]$. Let $\Omega$ be a bounded domain in a Carnot group, and let $v: \partial \Omega \rightarrow \mathbb{R}$ be a continuous function. Then the Dirichlet problem

$$
\begin{cases}\Delta_{\infty} u=0 & \text { in } \Omega, \\ u=v & \text { on } \partial \Omega\end{cases}
$$

has a unique viscosity solution $u_{\infty}$.

Our goal is to prove a parabolic version of Theorem 1.1 for a class of equations (defined in the next section), namely:

Conjecture 1.2. Let $\Omega$ be a bounded domain in a Carnot group, and let $T>0$. Let $\psi \in C(\bar{\Omega})$ and $g \in C(\Omega \times[0, T))$ Then the Cauchy-Dirichlet problem

$$
\begin{cases}u_{t}-\Delta_{\infty}^{h} u=0 & \text { in } \Omega \times(0, T), \\ u(x, 0)=\psi(x) & \text { on } \bar{\Omega}, \\ u(x, t)=g(x, t) & \text { on } \partial \Omega \times(0, T)\end{cases}
$$

has a unique viscosity solution $u$.

In Sections 2 and 3, we review key properties of Carnot groups and parabolic viscosity solutions. In Section 4, we prove the uniqueness, and Section 5 covers the existence.

\section{Calculus on Carnot Groups}

We begin by denoting an arbitrary Carnot group in $\mathbb{R}^{N}$ by $G$ and its corresponding Lie algebra by $g$. Recall that $g$ is nilpotent and stratified, resulting in the

Received January 26, 2015. Revision received June 23, 2016.

The first author was partially supported by a University of South Florida Proposal Enhancement Grant. 
decomposition

$$
g=V_{1} \oplus V_{2} \oplus \cdots \oplus V_{l}
$$

for appropriate vector spaces that satisfy the Lie bracket relation $\left[V_{1}, V_{j}\right]=V_{1+j}$. The Lie algebra $g$ is associated with the group $G$ via the exponential map exp : $g \rightarrow G$. Since this map is a diffeomorphism, we can choose a basis for $g$ so that it is the identity map. Denote this basis by

$$
X_{1}, X_{2}, \ldots, X_{n_{1}}, Y_{1}, Y_{2}, \ldots, Y_{n_{2}}, Z_{1}, Z_{2}, \ldots, Z_{n_{3}},
$$

so that

$$
\begin{aligned}
V_{1} & =\operatorname{span}\left\{X_{1}, X_{2}, \ldots, X_{n_{1}}\right\}, \\
V_{2} & =\operatorname{span}\left\{Y_{1}, Y_{2}, \ldots, Y_{n_{2}}\right\}, \\
V_{3} \oplus V_{4} \oplus \cdots \oplus V_{l} & =\operatorname{span}\left\{Z_{1}, Z_{2}, \ldots, Z_{n_{3}}\right\} .
\end{aligned}
$$

We endow $g$ with an inner product $\langle\cdot, \cdot\rangle$ and related norm $\|\cdot\|$ so that this basis is orthonormal. Clearly, the Riemannian dimension of $g$ (and so $G$ ) is $N=n_{1}+n_{2}+$ $n_{3}$. However, we will also consider the homogeneous dimension of $G$, denoted $\mathcal{Q}$, which is given by

$$
\mathcal{Q}=\sum_{i=1}^{l} i \cdot \operatorname{dim} V_{i}
$$

Before proceeding with the calculus, we recall the group and metric space properties. Since the exponential map is the identity, the group law is the Campbell-Hausdorff formula (see, e.g., [7]). For our purposes, this formula is given by

$$
p \cdot q=p+q+\frac{1}{2}[p, q]+R(p, q),
$$

where $R(p, q)$ are terms of order 3 or higher. The identity element of $G$ will be denoted by 0 and called the origin. There is also a natural metric on $G$, which is the Carnot-Carathéodory distance, defined for the points $p$ and $q$ as follows:

$$
d_{C}(p, q)=\inf _{\Gamma} \int_{0}^{1}\left\|\gamma^{\prime}(t)\right\| d t,
$$

where $\Gamma$ is the set of all curves $\gamma$ such that $\gamma(0)=p, \gamma(1)=q$, and $\gamma^{\prime}(t) \in V_{1}$. By Chow's theorem (see, e.g., [2]) any two points can be connected by such a curve, which means that $d_{C}(p, q)$ is an honest metric. Define the CarnotCarathéodory ball of radius $r$ centered at a point $p_{0}$ by

$$
B\left(p_{0}, r\right)=\left\{p \in G: d_{C}\left(p, p_{0}\right)<r\right\} .
$$

In addition to the Carnot-Carathéodory metric, there is a smooth (off the origin) gauge. This gauge is defined for a point $p=\left(\zeta_{1}, \zeta_{2}, \ldots, \zeta_{l}\right)$ with $\zeta_{i} \in V_{i}$ by

$$
\mathcal{N}(p)=\left(\sum_{i=1}^{l}\left\|\zeta_{i}\right\|^{2 l ! / i}\right)^{1 /(2 l !)}
$$


and induces the metric $d_{\mathcal{N}}$ that is bi-Lipschitz equivalent to the CarnotCarathéodory metric and is given by

$$
d_{\mathcal{N}}(p, q)=\mathcal{N}\left(p^{-1} \cdot q\right) .
$$

We define the gauge ball of radius $r$ centered at a point $p_{0}$ by

$$
B_{\mathcal{N}}\left(p_{0}, r\right)=\left\{p \in G: d_{\mathcal{N}}\left(p, p_{0}\right)<r\right\} .
$$

In this environment, a smooth function $u: G \rightarrow \mathbb{R}$ has the horizontal derivative given by

$$
\nabla_{0} u=\left(X_{1} u, X_{2} u, \ldots, X_{n_{1}} u\right)
$$

and the symmetrized horizontal second derivative matrix, denoted by $\left(D^{2} u\right)^{\star}$, with entries

$$
\left(\left(D^{2} u\right)^{\star}\right)_{i j}=\frac{1}{2}\left(X_{i} X_{j} u+X_{j} X_{i} u\right)
$$

for $i, j=1,2, \ldots, n_{1}$. We also consider the semihorizontal derivative given by

$$
\nabla_{1} u=\left(X_{1} u, X_{2} u, \ldots, X_{n_{1}} u, Y_{1} u, Y_{2} u, \ldots, Y_{n_{2}} u\right) .
$$

Using these derivatives, we define the $h$-homogeneous infinite Laplace operator for $h \geq 1$ by

$$
\Delta_{\infty}^{h} f=\left\|\nabla_{0} f\right\|^{h-3} \sum_{i, j=1}^{n_{1}} X_{i} f X_{j} f X_{i} X_{j} f=\left\|\nabla_{0} f\right\|^{h-3}\left\langle\left(D^{2} f\right)^{\star} \nabla_{0} f, \nabla_{0} f\right\rangle .
$$

Given $T>0$ and a function $u: G \times[0, T] \rightarrow \mathbb{R}$, we may define the analogous subparabolic infinite Laplace operator by

$$
u_{t}-\Delta_{\infty}^{h} u
$$

and we consider the corresponding equation

$$
u_{t}-\Delta_{\infty}^{h} u=0
$$

We note that when $h \geq 3$, this operator is continuous. When $h=3$, we have the subparabolic infinite Laplace equation analogous to the infinite Laplace operator in [5]. The Euclidean analog for $h=1$ has been explored in [12], and the Euclidean analog for $1<h<3$ in [13].

We recall that for any open set $\mathcal{O} \subset G$, the function $f$ is in the horizontal Sobolev space $W^{1, \mathrm{p}}(\mathcal{O})$ if $f$ and $X_{i} f$ are in $L^{\mathrm{p}}(\mathcal{O})$ for $i=1,2, \ldots, n_{1}$. Replacing $L^{\mathrm{p}}(\mathcal{O})$ by $L_{\text {loc }}^{\mathrm{p}}(\mathcal{O})$, the space $W_{\text {loc }}^{1, \mathrm{p}}(\mathcal{O})$ is defined similarly. The space $W_{0}^{1, \mathrm{p}}(\mathcal{O})$ is the closure in $W^{1, \mathrm{p}}(\mathcal{O})$ of smooth functions with compact support. In addition, we recall that a function $u: G \rightarrow \mathbb{R}$ is $\mathcal{C}_{\text {sub }}^{2}$ if $\nabla_{1} u$ and $X_{i} X_{j} u$ are continuous for all $i, j=1,2, \ldots n_{1}$. Note that $\mathcal{C}_{\text {sub }}^{2}$ is not equivalent to (Euclidean) $C^{2}$. For spaces involving time, the space $C\left(t_{1}, t_{2} ; X\right)$ consists of all continuous functions $u:\left[t_{1}, t_{2}\right] \rightarrow X$ with $\max _{t_{1} \leq t \leq t_{2}}\|u(\cdot, t)\|_{X}<\infty$. A similar definition holds for $L^{\mathrm{p}}\left(t_{1}, t_{2} ; X\right)$.

Given an open box $\mathcal{O}=\left(a_{1}, b_{1}\right) \times\left(a_{2}, b_{2}\right) \times \cdots \times\left(a_{N}, b_{N}\right)$, we define the parabolic space $\mathcal{O}_{t_{1}, t_{2}}$ to be $\mathcal{O} \times\left[t_{1}, t_{2}\right]$. Its parabolic boundary is given by $\partial_{\text {par }} \mathcal{O}_{t_{1}, t_{2}}=\left(\overline{\mathcal{O}} \times\left\{t_{1}\right\}\right) \cup\left(\partial \mathcal{O} \times\left(t_{1}, t_{2}\right]\right)$. 
Finally, recall that if $G$ is a Carnot group with homogeneous dimension $\mathcal{Q}$, then $G \times \mathbb{R}$ is again a Carnot group of homogeneous dimension $\mathcal{Q}+1$, where we have added an extra vector field $\frac{\partial}{\partial t}$ to the first layer of the grading. This allows us to give meaning to notations such as $W^{1,2}\left(\mathcal{O}_{t_{1}, t_{2}}\right)$ and $\mathcal{C}_{\text {sub }}^{2}\left(\mathcal{O}_{t_{1}, t_{2}}\right)$, where we consider $\nabla_{0} u$ to be $\left(X_{1} u, X_{2} u, \ldots, X_{n_{1}} u, \frac{\partial u}{\partial t}\right)$.

\section{Parabolic Jets and Viscosity Solutions}

\subsection{Parabolic Jets}

In this subsection, we recall the definitions of the parabolic jets, as given in [6], but included here for completeness. We define the parabolic superjet of $u(p, t)$ at the point $\left(p_{0}, t_{0}\right) \in \mathcal{O}_{t_{1}, t_{2}}$, denoted $P^{2,+} u\left(p_{0}, t_{0}\right)$, by using triples $(a, \eta, X) \in$ $\mathbb{R} \times V_{1} \oplus V_{2} \times S^{n_{1}}$ so that $(a, \eta, X) \in P^{2,+} u\left(p_{0}, t_{0}\right)$ if

$$
\begin{aligned}
u(p, t) \leq & u\left(p_{0}, t_{0}\right)+a\left(t-t_{0}\right)+\left\langle\eta, \widehat{p_{0}^{-1} \cdot p}\right\rangle+\frac{1}{2}\left\langle X \overline{p_{0}^{-1} \cdot p}, \overline{p_{0}^{-1} \cdot p}\right\rangle \\
& +o\left(\left|t-t_{0}\right|+\left|p_{0}^{-1} \cdot p\right|^{2}\right) \quad \text { as }(p, t) \rightarrow\left(p_{0}, t_{0}\right) .
\end{aligned}
$$

We recall that $S^{k}$ is the set of $k \times k$ symmetric matrices and $n_{i}=\operatorname{dim} V_{i}$. We define $\overline{p_{0}^{-1} \cdot p}$ as the first $n_{1}$ coordinates of $p_{0}^{-1} \cdot p$ and $\overline{p_{0}^{-1} \cdot p}$ as the first $n_{1}+n_{2}$ coordinates of $p_{0}^{-1} \cdot p$. This definition is an extension of the superjet definition for subparabolic equations in the Heisenberg group [4]. We define the subjet $P^{2,-} u\left(p_{0}, t_{0}\right)$ by

$$
P^{2,-} u\left(p_{0}, t_{0}\right)=-P^{2,+}(-u)\left(p_{0}, t_{0}\right) .
$$

We define the set-theoretic closure of the superjet, denoted $\bar{P}^{2,+} u\left(p_{0}, t_{0}\right)$, by requiring $(a, \eta, X) \in \bar{P}^{2,+} u\left(p_{0}, t_{0}\right)$ exactly when there is a sequence $\left(a_{n}, p_{n}, t_{n}\right.$, $\left.u\left(p_{n}, t_{n}\right), \eta_{n}, X_{n}\right) \rightarrow\left(a, p_{0}, t_{0}, u\left(p_{0}, t_{0}\right), \eta, X\right)$ with the triple $\left(a_{n}, \eta_{n}, X_{n}\right) \in$ $P^{2,+} u\left(p_{n}, t_{n}\right)$. A similar definition holds for the closure of the subjet.

We may also define jets using appropriate test functions. Given a function $u$ : $\mathcal{O}_{t_{1}, t_{2}} \rightarrow \mathbb{R}$, we consider the set $\mathcal{A} u\left(p_{0}, t_{0}\right)$ given by

$$
\begin{aligned}
\mathcal{A} u\left(p_{0}, t_{0}\right)= & \left\{\phi \in \mathcal{C}_{\text {sub }}^{2}\left(\mathcal{O}_{t_{1}, t_{2}}\right): u(p, t)-\phi(p, t) \leq u\left(p_{0}, t_{0}\right)-\phi\left(p_{0}, t_{0}\right)=0\right. \\
& \left.\forall(p, t) \in \mathcal{O}_{t_{1}, t_{2}}\right\},
\end{aligned}
$$

consisting of all test functions that touch $u$ from above at $\left(p_{0}, t_{0}\right)$. We define the set of all test functions that touch from below, denoted $\mathcal{B} u\left(p_{0}, t_{0}\right)$, similarly.

The following lemma relates the test functions to jets. The proof is identical to that of Lemma 3.1 in [4] but uses the (smooth) gauge $\mathcal{N}(p)$ instead of Euclidean distance.

\section{LEMMA 3.1.}

$$
P^{2,+} u\left(p_{0}, t_{0}\right)=\left\{\left(\phi_{t}\left(p_{0}, t_{0}\right), \nabla \phi\left(p_{0}, t_{0}\right),\left(D^{2} \phi\left(p_{0}, t_{0}\right)\right)^{\star}\right): \phi \in \mathcal{A} u\left(p_{0}, t_{0}\right)\right\} .
$$




\subsection{Jet Twisting}

We recall that the set $V_{1}=\operatorname{span}\left\{X_{1}, X_{2}, \ldots, X_{n_{1}}\right\}$, and notationally, we will always denote $n_{1}$ by $n$. The vectors $X_{i}$ at the point $p \in G$ can be written as

$$
X_{i}(p)=\sum_{j=1}^{N} a_{i j}(p) \frac{\partial}{\partial x_{j}},
$$

forming the $n \times N$ matrix $\mathbb{A}$ with smooth entries $\mathbb{A}_{i j}=a_{i j}(p)$. By linear independence of the $X_{i}, \mathbb{A}$ has rank $n$. Similarly,

$$
Y_{i}(p)=\sum_{j=1}^{N} b_{i j}(p) \frac{\partial}{\partial x_{j}},
$$

forming the $n_{2} \times N$ matrix $\mathbb{B}$ with smooth entries $\mathbb{B}_{i j}=b_{i j}$. The matrix $\mathbb{B}$ has rank $n_{2}$. The following lemma differs from [5, Cor. 3.2] only in that there is now a parabolic term. This term, however, does not need to be twisted. The proof is then identical since only the space terms need twisting.

Lemma 3.2. Let $(a, \eta, X) \in \bar{P}_{\text {eucl }}^{2,+} u(p, t)$. (Recall that $(\eta, X) \in \mathbb{R}^{N} \times S^{N}$.) Then

$$
\left(a, \mathbb{A} \cdot \eta \oplus \mathbb{B} \cdot \eta, \mathbb{A} X \mathbb{A}^{T}+\mathbb{M}\right) \in \bar{P}^{2,+} u(p, t) .
$$

Here the entries of the (symmetric) matrix $\mathbb{M}$ are given by

$$
\mathbb{M}_{i j}= \begin{cases}\sum_{k=1}^{N} \sum_{l=1}^{N}\left(a_{i l}(p) \frac{\partial}{\partial x_{l}} a_{j k}(p)+a_{j l}(p) \frac{\partial a_{i k}}{\partial x_{l}}(p)\right) \eta_{k}, & i \neq j, \\ \sum_{k=1}^{N} \sum_{l=1}^{N} a_{i l}(p) \frac{\partial a_{i k}}{\partial x_{l}}(p) \eta_{k}, & i=j .\end{cases}
$$

\subsection{Viscosity Solutions}

We consider parabolic equations of the form

$$
u_{t}+F\left(t, p, u, \nabla_{1} u,\left(D^{2} u\right)^{\star}\right)=0
$$

for continuous and proper $F:[0, T] \times G \times \mathbb{R} \times g \times S^{n} \rightarrow \mathbb{R}$ [8]. We recall that $S^{n}$ is the set of $n \times n$ symmetric matrices (where $\operatorname{dim} V_{1}=n$ ) and the derivatives $\nabla_{1} u$ and $\left(D^{2} u\right)^{\star}$ are taken in the space variable $p$. We then use the jets to define subsolutions and supersolutions to Equation (3.1) in the usual way.

Definition 1. Let $\left(p_{0}, t_{0}\right) \in \mathcal{O}_{t_{1}, t_{2}}$ be as before. The upper semicontinuous function $u$ is a parabolic viscosity subsolution in $\mathcal{O}_{t_{1}, t_{2}}$ if for all $\left(p_{0}, t_{0}\right) \in \mathcal{O}_{t_{1}, t_{2}}$, we have that $(a, \eta, X) \in \bar{P}^{2,+} u\left(p_{0}, t_{0}\right)$ produces

$$
a+F\left(t_{0}, p_{0}, u\left(p_{0}, t_{0}\right), \eta, X\right) \leq 0 .
$$

A lower semicontinuous function $u$ is a parabolic viscosity supersolution in $\mathcal{O}_{t_{1}, t_{2}}$ if for all $\left(p_{0}, t_{0}\right) \in \mathcal{O}_{t_{1}, t_{2}}$, we have that $(b, v, Y) \in \bar{P}^{2,-} u\left(p_{0}, t_{0}\right)$ produces

$$
b+F\left(t_{0}, p_{0}, u\left(p_{0}, t_{0}\right), v, Y\right) \geq 0 .
$$

A continuous function $u$ is a parabolic viscosity solution in $\mathcal{O}_{t_{1}, t_{2}}$ if it is both a parabolic viscosity subsolution and parabolic viscosity supersolution. 
REMARK 3.3. In the special case where $F\left(t, p, u, \nabla_{1} u,\left(D^{2} u\right)^{\star}\right)=F_{\infty}^{h}\left(\nabla_{0} u\right.$, $\left.\left(D^{2} u\right)^{\star}\right)=-\Delta_{\infty}^{h} u$ for $h \geq 3$, we use the terms "parabolic viscosity $h$-infinite supersolution", and so on.

In the case where $1 \leq h<3$, the definition above is insufficient due to the singularity occurring when the horizontal gradient vanishes. Therefore, following [12] and [13], we define viscosity solutions to Equation (2.3) when $1 \leq h<3$ as follows.

Definition 2. Let $\mathcal{O}_{t_{1}, t_{2}}$ be as before. A lower semicontinuous function $v$ : $\mathcal{O}_{t_{1}, t_{2}} \rightarrow \mathbb{R}$ is a parabolic viscosity $h$-infinite supersolution of $u_{t}-\Delta_{\infty}^{h} u=0$ if whenever $\left(p_{0}, t_{0}\right) \in \mathcal{O}_{t_{1}, t_{2}}$ and $\phi \in \mathcal{B} u\left(p_{0}, t_{0}\right)$, we have

$$
\begin{cases}\phi_{t}\left(p_{0}, t_{0}\right)-\Delta_{\infty}^{h} \phi\left(p_{0}, t_{0}\right) \geq 0 & \text { when } \nabla_{0} \phi\left(p_{0}, t_{0}\right) \neq 0, \\ \phi_{t}\left(p_{0}, t_{0}\right)-\min _{\|\eta\|=1}\left\langle\left(D^{2} \phi\right)^{\star}\left(p_{0}, t_{0}\right) \eta, \eta\right\rangle \geq 0 & \text { when } \nabla_{0} \phi\left(p_{0}, t_{0}\right)=0 \text { and } \\ \phi_{t}\left(p_{0}, t_{0}\right) \geq 0 & h=1, \\ & \text { when } \nabla_{0} \phi\left(p_{0}, t_{0}\right)=0 \text { and } \\ & 1<h<3 .\end{cases}
$$

An upper semicontinuous function $u: \mathcal{O}_{t_{1}, t_{2}} \rightarrow \mathbb{R}$ is a parabolic viscosity $h$ infinite subsolution of $u_{t}-\Delta_{\infty}^{h} u=0$ if whenever $\left(p_{0}, t_{0}\right) \in \mathcal{O}_{t_{1}, t_{2}}$ and $\phi \in$ $\mathcal{A} u\left(p_{0}, t_{0}\right)$, we have

$$
\begin{cases}\phi_{t}\left(p_{0}, t_{0}\right)-\Delta_{\infty}^{h} \phi\left(p_{0}, t_{0}\right) \leq 0 & \text { when } \nabla_{0} \phi\left(p_{0}, t_{0}\right) \neq 0, \\ \phi_{t}\left(p_{0}, t_{0}\right)-\max _{\|\eta\|=1}\left\langle\left(D^{2} \phi\right)^{\star}\left(p_{0}, t_{0}\right) \eta, \eta\right\rangle \leq 0 & \text { when } \nabla_{0} \phi\left(p_{0}, t_{0}\right)=0 \text { and } \\ \phi_{t}\left(p_{0}, t_{0}\right) \leq 0 & h=1, \\ & \text { when } \nabla_{0} \phi\left(p_{0}, t_{0}\right)=0 \text { and } \\ & 1<h<3 .\end{cases}
$$

A continuous function is a parabolic viscosity $h$-infinite solution if it is both a parabolic viscosity $h$-infinite subsolution and parabolic viscosity $h$-infinite subsolution.

REMARK 3.4. When $1<h<3$, we can actually consider the continuous operator

$$
\begin{aligned}
& F_{\infty}^{h}\left(\nabla_{0} u,\left(D^{2} u\right)^{\star}\right) \\
& \quad= \begin{cases}-\left\|\nabla_{0} u\right\|^{h-3}\left\langle\left(D^{2} u\right)^{\star} \nabla_{0} u, \nabla_{0} u\right\rangle=-\Delta_{\infty}^{h} u, & \nabla_{0} u \neq 0, \\
0, & \nabla_{0} u=0 .\end{cases}
\end{aligned}
$$

Definitions 1 and 2 would then agree (cf. [13]).

We also wish to define what [11] refers to as parabolic viscosity solutions. We first need to consider the set

$$
\begin{aligned}
\mathcal{A}^{-} u\left(p_{0}, t_{0}\right)= & \left\{\phi \in \mathcal{C}^{2}\left(\mathcal{O}_{t_{1}, t_{2}}\right): u(p, t)-\phi(p, t) \leq u\left(p_{0}, t_{0}\right)-\phi\left(p_{0}, t_{0}\right)=0\right. \\
& \text { for } \left.p \neq p_{0}, t<t_{0}\right\},
\end{aligned}
$$


consisting of all functions that touch from above only when $t<t_{0}$. Note that this set is larger than $\mathcal{A} u$ and corresponds physically to the past alone playing a role in determining the present. We define $\mathcal{B}^{-} u\left(p_{0}, t_{0}\right)$ similarly. We then have the following definition.

Definition 3. An upper semicontinuous function $u$ on $\mathcal{O}_{t_{1}, t_{2}}$ is a past parabolic viscosity subsolution in $\mathcal{O}_{t_{1}, t_{2}}$ if $\phi \in \mathcal{A}^{-} u\left(p_{0}, t_{0}\right)$ produces

$$
\phi_{t}\left(p_{0}, t_{0}\right)+F\left(t_{0}, p_{0}, u\left(p_{0}, t_{0}\right), \nabla_{1} \phi\left(p_{0}, t_{0}\right),\left(D^{2} \phi\left(p_{0}, t_{0}\right)\right)^{\star}\right) \leq 0 .
$$

An lower semicontinuous function $u$ on $\mathcal{O}_{t_{1}, t_{2}}$ is a past parabolic viscosity supersolution in $\mathcal{O}_{t_{1}, t_{2}}$ if $\phi \in \mathcal{B}^{-} u\left(p_{0}, t_{0}\right)$ produces

$$
\phi_{t}\left(p_{0}, t_{0}\right)+F\left(t_{0}, p_{0}, u\left(p_{0}, t_{0}\right), \nabla_{1} \phi\left(p_{0}, t_{0}\right),\left(D^{2} \phi\left(p_{0}, t_{0}\right)\right)^{\star}\right) \geq 0 .
$$

A continuous function is a past parabolic viscosity solution if it is both a past parabolic viscosity supersolution and subsolution.

We have the following proposition whose proof is obvious. The analogous theorem and its converse for the Euclidean case can be found in [11]. We will address the converse in the Carnot group case in the next section.

Proposition 3.5. Past parabolic viscosity sub(super)solutions are parabolic viscosity sub(super)solutions. In particular, past parabolic viscosity h-infinite sub(super)solutions are parabolic viscosity $h$-infinite sub(super)solutions for $h \geq 1$.

\subsection{The Carnot Parabolic Maximum Principle}

In this subsection, we recall the Carnot parabolic maximum principle and key corollaries, as proved in [6].

Lemma 3.6 (Carnot parabolic maximum principle). Let $u$ be a viscosity subsolution to Equation (3.1), and $v$ be a viscosity supersolution to Equation (3.1) in the bounded parabolic set $\Omega \times(0, T)$ where $\Omega$ is a (bounded) domain, and let $\tau$ be a positive real parameter. Let $\phi(p, q, t)=\varphi\left(p \cdot q^{-1}, t\right)$ be a $C^{2}$ function in the space variables $p$ and $q$, and a $C^{1}$ function in $t$. Suppose that the local maximum

$$
M_{\tau} \equiv \max _{\bar{\Omega} \times \bar{\Omega} \times[0, T]}\{u(p, t)-v(q, t)-\tau \phi(p, q, t)\}
$$

occurs at the interior point $\left(p_{\tau}, q_{\tau}, t_{\tau}\right)$ of the parabolic set $\Omega \times \Omega \times(0, T)$. Define the $n \times n$ matrix $W$ by

$$
W_{i j}=X_{i}(p) X_{j}(q) \phi\left(p_{\tau}, q_{\tau}, t_{\tau}\right) .
$$

Let the $2 n \times 2 n$ matrix $\mathfrak{W}$ be given by

$$
\mathfrak{W}=\left(\begin{array}{cc}
0 & \frac{1}{2}\left(W-W^{T}\right) \\
\frac{1}{2}\left(W^{T}-W\right) & 0
\end{array}\right),
$$


and let the matrix $\mathcal{W} \in S^{2 N}$ be given by

$$
\mathcal{W}=\left(\begin{array}{cc}
D_{p p}^{2} \phi\left(p_{\tau}, q_{\tau}, t_{\tau}\right) & D_{p q}^{2} \phi\left(p_{\tau}, q_{\tau}, t_{\tau}\right) \\
D_{q p}^{2} \phi\left(p_{\tau}, q_{\tau}, t_{\tau}\right) & D_{q q}^{2} \phi\left(p_{\tau}, q_{\tau}, t_{\tau}\right)
\end{array}\right) .
$$

Suppose that

$$
\lim _{\tau \rightarrow \infty} \tau \phi\left(p_{\tau}, q_{\tau}, t_{\tau}\right)=0 .
$$

Then for each $\tau>0$, there exists real numbers $a_{1}$ and $a_{2}$, symmetric matrices $\mathcal{X}_{\tau}$ and $\mathcal{Y}_{\tau}$, and a vector $\Upsilon_{\tau} \in V_{1} \oplus V_{2}$, namely $\Upsilon_{\tau}=\nabla_{1}(p) \phi\left(p_{\tau}, q_{\tau}, t_{\tau}\right)$, such that the following hold:

A) $\left(a_{1}, \tau \Upsilon_{\tau}, \mathcal{X}_{\tau}\right) \in \bar{P}^{2,+} u\left(p_{\tau}, t_{\tau}\right)$ and $\left(a_{2}, \tau \Upsilon_{\tau}, \mathcal{Y}_{\tau}\right) \in \bar{P}^{2,-} v\left(q_{\tau}, t_{\tau}\right)$.

B) $a_{1}-a_{2}=\phi_{t}\left(p_{\tau}, q_{\tau}, t_{\tau}\right)$.

C) For any vectors $\xi, \epsilon \in V_{1}$, we have

$$
\begin{aligned}
\left\langle\mathcal{X}_{\tau} \xi, \xi\right\rangle-\left\langle\mathcal{Y}_{\tau} \epsilon, \epsilon\right\rangle \leq & \tau\left\langle\left(D_{p}^{2} \phi\right)^{\star}\left(p_{\tau}, q_{\tau}, t_{\tau}\right)(\xi-\epsilon),(\xi-\epsilon)\right\rangle \\
& +\tau\langle\mathfrak{W}(\xi \oplus \epsilon),(\xi \oplus \epsilon)\rangle \\
& +\tau\|\mathcal{W}\|^{2}\left\|\mathbb{A}(\hat{p})^{T} \xi \oplus \mathbb{A}(\hat{q})^{T} \epsilon\right\|^{2} .
\end{aligned}
$$

In particular,

$$
\left\langle\mathcal{X}_{\tau} \xi, \xi\right\rangle-\left\langle\mathcal{Y}_{\tau} \xi, \xi\right\rangle \lesssim \tau\|\mathcal{W}\|^{2}\|\xi\|^{2}
$$

Corollary 3.7. Let $\phi(p, q, t)=\phi(p, q)=\varphi\left(p \cdot q^{-1}\right)$ be a nonnegative function independent of $t$. Suppose that $\phi(p, q)=0$ exactly when $p=q$. Then

$$
\lim _{\tau \rightarrow \infty} \tau \phi\left(p_{\tau}, q_{\tau}\right)=0
$$

In particular, if

$$
\phi(p, q, t)=\frac{1}{m} \sum_{i=1}^{N}\left(\left(p \cdot q^{-1}\right)_{i}\right)^{m}
$$

for some even integer $m \geq 4$ where $\left(p \cdot q^{-1}\right)_{i}$ is the ith component of the Carnot group multiplication group law, then for the vector $\Upsilon_{\tau}$ and matrices $\mathcal{X}_{\tau}, \mathcal{Y}_{\tau}$ from the lemma, we have:

A) $\left(a_{1}, \tau \Upsilon_{\tau}, \mathcal{X}_{\tau}\right) \in \bar{P}^{2,+} u\left(p_{\tau}, t_{\tau}\right)$ and $\left(a_{1}, \tau \Upsilon_{\tau}, \mathcal{Y}_{\tau}\right) \in \bar{P}^{2,-} v\left(q_{\tau}, t_{\tau}\right)$.

B) The vector $\Upsilon_{\tau}$ satisfies

$$
\left\|\Upsilon_{\tau}\right\| \sim \phi\left(p_{\tau}, q_{\tau}\right)^{(m-1) / m}
$$

C) For any fixed vector $\xi \in V_{1}$, we have

$$
\left\langle\mathcal{X}_{\tau} \xi, \xi\right\rangle-\left\langle\mathcal{Y}_{\tau} \xi, \xi\right\rangle \lesssim \tau\|\mathcal{W}\|^{2}\|\xi\|^{2} \lesssim \tau\left(\phi\left(p_{\tau}, q_{\tau}\right)\right)^{(2 m-4) / m}\|\xi\|^{2} .
$$




\section{Uniqueness of Viscosity Solutions}

We wish to formulate a comparison principle for the following problem.

Problem 4.1. Let $h \geq 1$. Let $\Omega$ be a bounded domain, and let $\Omega_{T}=\Omega \times[0, T)$. Let $\psi \in C(\bar{\Omega})$ and $g \in C\left(\overline{\Omega_{T}}\right)$. We consider the following boundary and initial value problem:

$$
\begin{cases}u_{t}+F_{\infty}^{h}\left(\nabla_{0} u,\left(D^{2} u\right)^{\star}\right)=0 & \text { in } \Omega \times(0, T), \quad(\mathrm{E}) \\ u(p, t)=g(p, t), & p \in \partial \Omega, t \in[0, T), \quad(\mathrm{BC}) \\ u(p, 0)=\psi(p), & p \in \overline{\Omega .} \quad \text { (IC) }\end{cases}
$$

We also adopt the definition that a subsolution $u(p, t)$ to Problem 4.1 is a viscosity subsolution to $(\mathrm{E}), u(p, t) \leq g(p, t)$ on $\partial \Omega$ with $0 \leq t<T$, and $u(p, 0) \leq \psi(p)$ on $\bar{\Omega}$. Supersolutions and solutions are defined in an analogous matter.

Because our solution $u$ will be continuous, we offer the following remark.

REMARK 4.2. The functions $\psi$ and $g$ may be replaced by one function $g \in C\left(\overline{\Omega_{T}}\right)$. This combines conditions (E) and (BC) into one condition

$$
u(p, t)=g(p, t), \quad(p, t) \in \partial_{\mathrm{par}} \Omega_{T} . \quad(\mathrm{IBC})
$$

TheOrem 4.3. Let $\Omega$ be a bounded domain in $G$, and let $h \geq 1$. If $u$ is a parabolic viscosity subsolution and $v$ a parabolic viscosity supersolution to Problem 4.1, then $u \leq v$ on $\Omega_{T} \equiv \Omega \times[0, T)$.

Proof. Our proof follows that of [8, Thm. 8.2], and so we discuss only the main parts.

For $\varepsilon>0$, we substitute $\tilde{u}=u-\frac{\varepsilon}{T-t}$ for $u$ and prove the theorem for

$$
\begin{aligned}
& u_{t}+F_{\infty}^{h}\left(\nabla_{0} u,\left(D^{2} u\right)^{\star}\right) \leq-\frac{\varepsilon}{T^{2}}<0, \\
& \lim _{t \uparrow T} u(p, t)=-\infty \quad \text { uniformly on } \bar{\Omega},
\end{aligned}
$$

and take limits to obtain the desired result. Assume that the maximum occurs at $\left(p_{0}, t_{0}\right) \in \Omega \times(0, T)$ with

$$
u\left(p_{0}, t_{0}\right)-v\left(p_{0}, t_{0}\right)=\delta>0 .
$$

Case 1: $h>1$.

Let $H \geq h+3$ be an even number. As in Equation (3.8), we let

$$
\phi(p, q)=\frac{1}{H} \sum_{i=1}^{N}\left(\left(p \cdot q^{-1}\right)_{i}\right)^{H}
$$

where $\left(p \cdot q^{-1}\right)_{i}$ is the $i$ th component of the Carnot group multiplication group law. Let

$$
M_{\tau}=u\left(p_{\tau}, t_{\tau}\right)-v\left(q_{\tau}, t_{\tau}\right)-\tau \phi\left(p_{\tau}, q_{\tau}\right)
$$


with $\left(p_{\tau}, q_{\tau}, t_{\tau}\right)$ the maximum point in $\bar{\Omega} \times \bar{\Omega} \times[0, T)$ of $u(p, t)-v(q, t)-$ $\tau \phi(p, q)$.

If $t_{\tau}=0$, then we have

$$
0<\delta \leq M_{\tau} \leq \sup _{\bar{\Omega} \times \bar{\Omega}}(\psi(p)-\psi(q)-\tau \phi(p, q)),
$$

leading to a contradiction for large $\tau$. We therefore conclude that $t_{\tau}>0$ for large $\tau$. Since $u \leq v$ on $\partial \Omega \times[0, T)$ by Equation (BC) of Problem 4.1, we conclude that for large $\tau$, we have that $\left(p_{\tau}, q_{\tau}, t_{\tau}\right)$ is an interior point, that is, $\left(p_{\tau}, q_{\tau}, t_{\tau}\right) \in \Omega \times \Omega \times(0, T)$. Using Corollary 3.7, Property A, we obtain

$$
\begin{aligned}
\left(a, \tau \Upsilon\left(p_{\tau}, q_{\tau}\right), \mathcal{X}_{\tau}\right) & \in \bar{P}^{2,+} u\left(p_{\tau}, t_{\tau}\right) \\
\text { and } \quad\left(a, \tau \Upsilon\left(p_{\tau}, q_{\tau}\right), \mathcal{Y}_{\tau}\right) & \in \bar{P}^{2,-} v\left(q_{\tau}, t_{\tau}\right),
\end{aligned}
$$

satisfying the equations

$$
\begin{aligned}
& a+F_{\infty}^{h}\left(\tau \Upsilon\left(p_{\tau}, q_{\tau}\right), \mathcal{X}_{\tau}\right) \leq-\frac{\varepsilon}{T^{2}} \\
\text { and } \quad & a+F_{\infty}^{h}\left(\tau \Upsilon\left(p_{\tau}, q_{\tau}\right), \mathcal{Y}_{\tau}\right) \geq 0 .
\end{aligned}
$$

If there is a subsequence $\left\{p_{\tau}, q_{\tau}\right\}_{\tau>0}$ such that $p_{\tau} \neq q_{\tau}$, we subtract, and using Corollary 3.7 , we have

$$
\begin{aligned}
0< & \frac{\varepsilon}{T^{2}} \\
\leq & \left(\tau \Upsilon\left(p_{\tau}, q_{\tau}\right)\right)^{h-3} \tau^{2}\left(\left\langle\mathcal{X}_{\tau} \Upsilon\left(p_{\tau}, q_{\tau}\right), \Upsilon\left(p_{\tau}, q_{\tau}\right)\right\rangle\right. \\
& \left.-\left\langle\mathcal{Y}_{\tau} \Upsilon\left(p_{\tau}, q_{\tau}\right), \Upsilon\left(p_{\tau}, q_{\tau}\right)\right\rangle\right) \\
\lesssim & \tau^{h}\left(\varphi\left(p_{\tau}, q_{\tau}\right)^{(H-1) / H}\right)^{h-3}\left(\varphi\left(p_{\tau}, q_{\tau}\right)\right)^{(2 H-4) / H}\left(\varphi\left(p_{\tau}, q_{\tau}\right)\right)^{(2 H-2) / H} \\
= & \tau^{h}\left(\varphi\left(p_{\tau}, q_{\tau}\right)\right)^{(H h+H-h-3) / H}=\left(\tau \varphi\left(p_{\tau}, q_{\tau}\right)\right)^{h} \varphi\left(p_{\tau}, q_{\tau}\right)^{(H-h-3) / H} .
\end{aligned}
$$

Because $H>h+3$, we arrive at a contradiction as $\tau \rightarrow \infty$.

If we have $p_{\tau}=q_{\tau}$, then we arrive at a contradiction since

$$
F_{\infty}^{h}\left(\tau \Upsilon\left(p_{\tau}, q_{\tau}\right), \mathcal{X}_{\tau}\right)=F_{\infty}^{h}\left(\tau \Upsilon\left(p_{\tau}, q_{\tau}\right), \mathcal{Y}_{\tau}\right)=0
$$

Case 2: $h=1$.

We follow the proof of Theorem 3.1 in [12]. Let

$$
\varphi(p, q, t, s)=\frac{1}{4} \sum_{i=1}^{N}\left(\left(p \cdot q^{-1}\right)_{i}\right)^{4}+\frac{1}{2}(t-s)^{2},
$$

and let $\left(p_{\tau}, q_{\tau}, t_{\tau}, s_{\tau}\right)$ be the maximum of

$$
u(p, t)-v(q, s)-\tau \phi(p, q, t, s) .
$$

Again, for large $\tau$, this point is an interior point. If we have a sequence where $p_{\tau} \neq q_{\tau}$, then Lemma 3.2 yields

$$
\begin{aligned}
&\left(\tau\left(t_{\tau}-s_{\tau}\right), \tau \Upsilon\left(p_{\tau}, q_{\tau}\right), \mathcal{X}_{\tau}\right) \in \bar{P}^{2,+} u\left(p_{\tau}, t_{\tau}\right) \\
& \text { and } \quad\left(\tau\left(t_{\tau}-s_{\tau}\right), \tau \Upsilon\left(p_{\tau}, q_{\tau}\right), \mathcal{Y}_{\tau}\right) \in \bar{P}^{2,-} v\left(q_{\tau}, s_{\tau}\right),
\end{aligned}
$$


satisfying the equations

$$
\begin{aligned}
& \tau\left(t_{\tau}-s_{\tau}\right)+F_{\infty}^{h}\left(\tau \Upsilon\left(p_{\tau}, q_{\tau}\right), \mathcal{X}_{\tau}\right) \leq-\frac{\varepsilon}{T^{2}} \\
& \text { and } \quad \tau\left(t_{\tau}-s_{\tau}\right)+F_{\infty}^{h}\left(\tau \Upsilon\left(p_{\tau}, q_{\tau}\right), \mathcal{Y}_{\tau}\right) \geq 0
\end{aligned}
$$

As in the first case, we subtract to obtain

$$
\begin{aligned}
0 & <\frac{\varepsilon}{T^{2}} \\
& \leq\left(\tau \Upsilon\left(p_{\tau}, q_{\tau}\right)\right)^{-2} \tau^{2}\left(\left\langle\mathcal{X}_{\tau} \Upsilon\left(p_{\tau}, q_{\tau}\right), \Upsilon\left(p_{\tau}, q_{\tau}\right)\right\rangle-\left\langle\mathcal{Y}_{\tau} \Upsilon\left(p_{\tau}, q_{\tau}\right), \Upsilon\left(p_{\tau}, q_{\tau}\right)\right\rangle\right) \\
& \lesssim \varphi\left(p_{\tau}, q_{\tau}\right)^{-3 / 2}\left(\tau \varphi\left(p_{\tau}, q_{\tau}\right) \varphi\left(p_{\tau}, q_{\tau}\right)^{3 / 2}\right)=\tau \varphi\left(p_{\tau}, q_{\tau}\right) .
\end{aligned}
$$

We arrive at a contradiction as $\tau \rightarrow \infty$.

If $p_{\tau}=q_{\tau}$, then $v(q, s)-\beta^{v}(q, s)$ has a local minimum at $\left(q_{\tau}, s_{\tau}\right)$ where

$$
\beta^{v}(q, s)=-\frac{\tau}{4} \sum_{i=1}^{N}\left(\left(p_{\tau} \cdot q^{-1}\right)_{i}\right)^{4}-\frac{\tau}{2}\left(t_{\tau}-s\right)^{2} .
$$

We then have

$$
0<\varepsilon\left(T-s_{\tau}\right)^{-2} \leq \beta_{s}^{v}\left(q_{\tau}, s_{\tau}\right)-\min _{\|\eta\|=1}\left\langle\left(D^{2} \beta^{v}\right)^{\star}\left(q_{\tau}, s_{\tau}\right) \eta, \eta\right\rangle .
$$

Similarly, $u(p, t)-\beta^{u}(p, t)$ has a local maximum at $\left(p_{\tau}, t_{\tau}\right)$ where

$$
\beta^{u}(p, t)=\frac{\tau}{4} \sum_{i=1}^{N}\left(\left(p \cdot q_{\tau}^{-1}\right)_{i}\right)^{4}+\frac{\tau}{2}\left(t-s_{\tau}\right)^{2} .
$$

We then have

$$
0 \geq \beta_{t}^{u}\left(p_{\tau}, t_{\tau}\right)-\max _{\|\eta\|=1}\left\langle\left(D^{2} \beta^{u}\right)^{\star}\left(p_{\tau}, t_{\tau}\right) \eta, \eta\right\rangle,
$$

and subtraction gives us

$$
\begin{aligned}
0< & \varepsilon\left(T-s_{\tau}\right)^{-2} \\
\leq & \max _{\|\eta\|=1}\left\langle\left(D^{2} \beta^{u}\right)^{\star}\left(p_{\tau}, t_{\tau}\right) \eta, \eta\right\rangle-\min _{\|\eta\|=1}\left\langle\left(D^{2} \beta^{v}\right)^{\star}\left(q_{\tau}, s_{\tau}\right) \eta, \eta\right\rangle \\
& +\beta_{s}^{v}\left(q_{\tau}, s_{\tau}\right)-\beta_{t}^{u}\left(p_{\tau}, t_{\tau}\right) \\
= & \tau \max _{\|\eta\|=1}\left\langle\left(D_{p p}^{2} \varphi\left(p \cdot q_{\tau}^{-1}\right)\right)^{\star}\left(p_{\tau}, t_{\tau}\right) \eta, \eta\right\rangle \\
& \quad-\tau \min _{\|\eta\|=1}\left\langle\left(D_{q q}^{2} \varphi\left(p_{\tau} \cdot q^{-1}\right)\right)^{\star}\left(q_{\tau}, s_{\tau}\right) \eta, \eta\right\rangle \\
& \quad+\tau\left(t_{\tau}-s_{\tau}\right)-\tau\left(t_{\tau}-s_{\tau}\right) \\
= & 0 .
\end{aligned}
$$

Here, the last equality comes from the fact that $p_{\tau}=q_{\tau}$ and from the definition of $\varphi\left(p \cdot q^{-1}\right)$.

The comparison principle has the following consequences concerning properties of solutions. 
COROLlary 4.4. Let $h \geq 1$. The past parabolic viscosity $h$-infinite solutions are exactly the parabolic viscosity $h$-infinite solutions.

Proof. By Proposition 3.5 past parabolic viscosity $h$-infinite sub(super)solutions are parabolic viscosity $h$-infinite sub(super)solutions. To prove the converse, we will follow the proof of the subsolution case found in [11], highlighting the main details. Assume that $u$ is not a past parabolic viscosity $h$-infinite subsolution. Let $\phi \in \mathcal{A}^{-} u\left(p_{0}, t_{0}\right)$ have the property that

$$
\phi_{t}\left(p_{0}, t_{0}\right)-\Delta_{\infty}^{h} \phi\left(p_{0}, t_{0}\right) \geq \epsilon>0
$$

for a small parameter $\epsilon$. We may assume that $p_{0}$ is the origin. Let $r>0$ and define $S_{r}=B_{\mathcal{N}}(r) \times\left(t_{0}-r, t_{0}\right)$, and let $\partial S_{r}$ be its parabolic boundary. Then the function

$$
\tilde{\phi}_{r}(p, t)=\phi(p, t)+\left(t_{0}-t\right)^{8 l !}-r^{8 l !}+(\mathcal{N}(p))^{8 l !}
$$

is a classical supersolution for sufficiently small $r$. We then observe that $u \leq \tilde{\phi}_{r}$ on $\partial S_{r}$ but $u\left(0, t_{0}\right)>\tilde{\phi}\left(0, t_{0}\right)$. Thus, the comparison principle, Theorem 4.3 , does not hold. Thus, $u$ is not a parabolic viscosity $h$-infinite subsolution. The supersolution case is identical and omitted.

The following corollary has a proof similar to that of [12, Lemma 3.2].

Corollary 4.5. Let $u: \Omega_{T} \rightarrow \mathbb{R}$ be upper semicontinuous. Let $\phi \in \mathcal{A} u\left(p_{0}, t_{0}\right)$. If

$$
\begin{cases}\phi_{t}\left(p_{0}, t_{0}\right)-\Delta_{\infty}^{1} \phi\left(p_{0}, t_{0}\right) \leq 0 & \text { when } \nabla_{0} \phi\left(p_{0}, t_{0}\right) \neq 0 \\ \phi_{t}\left(p_{0}, t_{0}\right) \leq 0 & \text { when } \nabla_{0} \phi\left(p_{0}, t_{0}\right)=0 \\ & \left(D^{2} \phi\right)^{\star}\left(p_{0}, t_{0}\right)=0,\end{cases}
$$

then $u$ is a viscosity subsolution to (E) of Problem 4.1.

We also have the following function estimates with respect to boundary data.

COROLlary 4.6. Let $h \geq 1$. Let $g_{1}, g_{2} \in C\left(\overline{\Omega_{T}}\right)$ and $u_{1}, u_{2}$ be parabolic viscosity solutions to Equation (4.1) with boundary data $g_{1}$ and $g_{2}$, respectively. Then

$$
\sup _{(p, t) \in \Omega_{T}}\left|u_{1}(p, t)-u_{2}(p, t)\right| \leq \sup _{(p, t) \in \partial_{\mathrm{par}} \Omega_{T}}\left|g_{1}(p, t)-g_{2}(p, t)\right| .
$$

Proof. The function $u^{+}(p, t)=u_{2}(p, t)+\sup _{(p, t) \in \partial_{\mathrm{par}} \Omega_{T}}\left|g_{1}(p, t)-g_{2}(p, t)\right|$ is a parabolic viscosity supersolution with boundary data $g_{1}$, and the function $u^{-}(p, t)=u_{2}(p, t)-\sup _{(p, t) \in \partial_{\mathrm{par}} \Omega_{T}}\left|g_{1}(p, t)-g_{2}(p, t)\right|$ is a parabolic viscosity subsolution with boundary data $g_{1}$. Moreover, $u^{-} \leq u_{1} \leq u^{+}$on $\partial_{\mathrm{par}} \Omega_{T}$, and by Theorem $4.3 u^{-} \leq u_{1} \leq u^{+}$in $\Omega_{T}$.

Corollary 4.7. Let $h \geq 1$. Let $g \in C\left(\overline{\Omega_{T}}\right)$. Then every parabolic viscosity solution to Problem 4.1 satisfies

$$
\sup _{(p, t) \in \Omega_{T}}|u(p, t)| \leq \sup _{(p, t) \in \partial_{\mathrm{par}} \Omega_{T}}|g(p, t)| .
$$


Proof. The proof is similar to the previous corollary but using the functions $u^{ \pm}(p, t)= \pm \sup _{(p, t) \in \partial_{\mathrm{par}} \Omega_{T}}|g(p, t)|$ instead.

\section{Existence of Viscosity Solutions}

\subsection{Parabolic Viscosity Infinite Solutions: The Continuity Case}

As before, we will focus on the equations of the form (3.1) for continuous and proper $F:[0, T] \times G \times \mathbb{R} \times g \times S^{n_{1}} \rightarrow \mathbb{R}$ that possess a comparison principle such as Theorem 4.3 or [6, Thm. 3.6]. We will use Perron's method combined with the Carnot parabolic maximum principle to yield the desired existence theorem. In particular, the following proofs are similar to those found in [10, Chap. 2] except that the Euclidean derivatives have been replaced with horizontal derivatives and the Euclidean norms have been replaced with the gauge norm.

Lemma 5.1. Let $\mathcal{L}$ be a collection of parabolic viscosity supersolutions to (3.1), and let $u(p, t)=\inf \{v(p, t): v \in \mathcal{L}\}$. If $u$ is finite in a dense subset of $\Omega_{T}=$ $\Omega \times[0, T)$, then $u$ is a parabolic viscosity supersolution to (3.1).

Proof. First, note that $u$ is lower semicontinuous since every $v \in \mathcal{L}$ is. Let $\left(p_{0}, t_{0}\right) \in \Omega_{T}$ and $\phi \in \mathcal{A} u\left(p_{0}, t_{0}\right)$. Now let

$$
\psi(p, t)=\phi(p, t)-\left(d_{\mathcal{N}}\left(p_{0}, p\right)\right)^{2 l !}-\left|t-t_{0}\right|^{2}
$$

and notice that $\psi \in \mathcal{A} u\left(p_{0}, t_{0}\right)$. Then

$$
\begin{aligned}
(u-\psi)(p, t)-\left(d_{\mathcal{N}}\left(p_{0}, p\right)\right)^{2 l !}-\left|t-t_{0}\right|^{2} & =(u-\phi)(p, t) \\
& \geq(u-\phi)\left(p_{0}, t_{0}\right) \\
& =(u-\psi)\left(p_{0}, t_{0}\right) \\
& =0
\end{aligned}
$$

yields

$$
(u-\psi)(p, t) \geq\left(d_{\mathcal{N}}\left(p_{0}, p\right)\right)^{2 l !}+\left|t-t_{0}\right|^{2} .
$$

Since $u$ is lower semicontinuous, there exists a sequence $\left\{\left(p_{k}, t_{k}\right)\right\}$ with $t_{k}<t_{0}$ converging to $\left(p_{0}, t_{0}\right)$ as $k \rightarrow \infty$ such that

$$
(u-\psi)\left(p_{k}, t_{k}\right) \rightarrow(u-\psi)\left(p_{0}, t_{0}\right)=0 .
$$

Since $u(p, t)=\inf \{v(p, t): v \in \mathcal{L}\}$, there exists a sequence $\left\{v_{k}\right\} \subset \mathcal{L}$ such that $v_{k}\left(p_{k}, t_{k}\right)<u\left(p_{k}, t_{k}\right)+1 / k$ for $k=1,2, \ldots$. Since $v_{k} \geq u$, Equation (5.1) gives us

$$
\left(v_{k}-\psi\right)(p, t) \geq(u-\psi)(p, t) \geq\left(d_{\mathcal{N}}\left(p_{0}, p\right)\right)^{2 l !}+\left|t-t_{0}\right|^{2} .
$$

Let $B \subset \Omega$ denote a compact neighborhood of $\left(p_{0}, t_{0}\right)$. Since $v_{k}-\psi$ is lower semicontinuous, it attains a minimum in $B$ at a point $\left(q_{k}, s_{k}\right) \in B$. Then by (5.1) and (5.2) we have

$$
\begin{aligned}
(u-\psi)\left(p_{k}, t_{k}\right)+1 / k & >\left(v_{k}-\psi\right)\left(p_{k}, t_{k}\right) \geq\left(v_{k}-\psi\right)\left(q_{k}, s_{k}\right) \\
& \geq\left(d_{\mathcal{N}}\left(p_{0}, q_{k}\right)\right)^{2 l !}+\left|s_{k}-t_{0}\right|^{2} \geq 0
\end{aligned}
$$


for sufficiently large $k$ such that $\left(p_{k}, t_{k}\right) \in B$. By the squeeze theorem, $\left(q_{k}, s_{k}\right) \rightarrow$ $\left(p_{0}, t_{0}\right)$ as $k \rightarrow \infty$. Let $\eta=\psi-\left(d_{\mathcal{N}}\left(q_{k}, p\right)\right)^{2 l !}-\left|s_{k}-t\right|^{2}$. Then $\eta \in \mathcal{A} v_{k}\left(q_{k}, s_{k}\right)$, and we have that

$$
\eta_{t}\left(q_{k}, s_{k}\right)+F\left(s_{k}, q_{k}, v_{k}\left(q_{k}, s_{k}\right), \nabla_{1} \eta\left(q_{k}, s_{k}\right),\left(D^{2} \eta\left(q_{k}, s_{k}\right)\right)^{\star}\right) \geq 0 .
$$

This implies

$$
\psi_{t}\left(q_{k}, s_{k}\right)+F\left(s_{k}, q_{k}, v_{k}\left(q_{k}, s_{k}\right), \nabla_{1} \psi\left(q_{k}, s_{k}\right),\left(D^{2} \psi\left(s_{k}, s_{k}\right)\right)^{\star}\right) \geq 0 .
$$

Letting $k \rightarrow \infty$ yields

$$
\phi_{t}\left(p_{0}, t_{0}\right)+F\left(t_{0}, p_{0}, u\left(p_{0}, t_{0}\right) \nabla_{1} \phi\left(p_{0}, t_{0}\right),\left(D^{2} \phi\left(p_{0}, t_{0}\right)\right)^{\star}\right) \geq 0
$$

and that $u$ is a parabolic viscosity supersolution, as desired.

A similar argument yields the following:

LEMMA 5.2. Let $\mathcal{L}$ be a collection of parabolic viscosity subsolutions to (3.1), and let $u(p, t)=\sup \{v(p, t): v \in \mathcal{L}\}$. If $u$ is finite in a dense subset of $\Omega_{T}$, then $u$ is a parabolic viscosity subsolution to (3.1).

For the following lemmas, we need to recall the following definition.

Definition 4. The upper and lower semicontinuous envelopes of a function $u$ are given by

$$
u^{*}(p, t):=\lim _{r \downarrow 0} \sup \left\{u(q, s):\left|q^{-1} p\right|_{g}+|s-t| \leq r\right\}
$$

and

$$
u_{*}(p, t):=\liminf _{r \downarrow 0}\left\{u(q, s):\left|q^{-1} p\right|_{g}+|s-t| \leq r\right\},
$$

respectively.

Lemma 5.3. Let $h$ be a parabolic viscosity supersolution to (3.1) in $\Omega_{T}$. Let $\mathcal{S}$ be the collection of all parabolic viscosity subsolutions $v$ of (3.1) satisfying $v \leq h$. If for $\hat{v} \in \mathcal{S}, \hat{v}_{*}$ is not a parabolic viscosity supersolution of (3.1), then there are a function $w \in \mathcal{S}$ and a point $\left(p_{0}, t_{0}\right)$ such that $\hat{v}\left(p_{0}, t_{0}\right)<w\left(p_{0}, t_{0}\right)$.

Proof. Let $\hat{v} \in \mathcal{S}$ such that $\hat{v}_{*}$ is not a parabolic viscosity supersolution of (3.1). Then there exist $(\hat{p}, \hat{t}) \in \Omega_{T}$ and $\phi \in \mathcal{A} \hat{v}_{*}(\hat{p}, \hat{t})$ such that

$$
\phi_{t}(p, t)+F\left(t, p, \hat{v}_{*}(p, t), \nabla_{1} \phi(p, t),\left(D^{2} \phi(p, t)\right)^{\star}\right)>0 .
$$

Let

$$
\psi(p, t)=\phi(p, t)-\left(d_{\mathcal{N}}(\hat{p}, p)\right)^{2 l !}-|t-\hat{t}|^{2}
$$

and notice that $\psi \in \mathcal{A} \hat{v}_{*}(\hat{p}, \hat{t})$. As in Lemma 5.1,

$$
\left(\hat{v}_{*}-\psi\right)(p, t) \geq\left(d_{\mathcal{N}}(\hat{p}, p)\right)^{2 l !}+|t-\hat{t}|^{2} .
$$

Let $B$ denote a compact neighborhood of $(\hat{p}, \hat{t})$, and let

$$
B_{k \epsilon}=B \cap\left\{(p, t):\left(d_{\mathcal{N}}(\hat{p}, p)\right)^{2 l !} \leq k \epsilon \text { and }|t-\hat{t}|^{2} \leq k \epsilon\right\} .
$$


Since $\hat{v} \in \mathcal{S}$, we have that $\hat{v} \leq h$, and thus $\psi(\hat{p}, \hat{t})=\hat{v}_{*}(\hat{p}, \hat{t}) \leq \hat{v}(\hat{p}, \hat{t}) \leq h(\hat{p}, \hat{t})$. However, if $\psi(\hat{p}, \hat{t})=h(\hat{p}, \hat{t})$, then $\psi \in \mathcal{A} h(\hat{p}, \hat{t})$, and inequality (5.3) would be contradictory. Thus,

$$
\psi(\hat{p}, \hat{t})<h(\hat{p}, \hat{t}) .
$$

Since $\psi$ is continuous and $h$ is lower semicontinuous, there exists $\epsilon>0$ such that

$$
\psi(p, t)+4 \epsilon \leq h(p, t)
$$

for $(p, t) \in B_{2 \epsilon}$. Notice that $\psi+4 \epsilon$ is a subsolution of (3.1) on the interior of $B_{2 \epsilon}$. Further, by (5.4)

$$
\hat{v}(p, t) \geq \hat{v}_{*}(p, t) \geq \psi(p, t)+4 \epsilon \text { for }(p, t) \in B_{2 \epsilon} \backslash B_{\epsilon} .
$$

We now define $\omega$ by

$$
\omega= \begin{cases}\max \{\psi(p, t)+4 \epsilon, \hat{v}(p, t)\}, & (p, t) \in B_{\epsilon}, \\ \hat{v}(p, t), & (p, t) \in \Omega_{T} \backslash B_{\epsilon} .\end{cases}
$$

But by (5.5)

$$
\omega(p, t)=\max \{\psi(p, t)+4 \epsilon, \hat{v}(p, t)\} \quad \text { for }(p, t) \in B_{2 \epsilon},
$$

not just for $(p, t) \in B_{\epsilon}$. Then by Lemma 5.2, $\omega$ is a subsolution in the interior of $B_{2 \epsilon}$ and thus a subsolution in $\Omega_{T}$. Therefore, $\omega \in \mathcal{S}$. Since

$$
0=\left(\hat{v}_{*}-\psi\right)(\hat{p}, \hat{t})=\liminf _{r \downarrow 0}\left\{(\hat{v}-\psi)(p, t):(p, t) \in B_{r}\right\},
$$

there is a point $\left(p_{0}, t_{0}\right) \in B_{\epsilon}$ that satisfies

$$
\hat{v}\left(p_{0}, t_{0}\right)-\psi\left(p_{0}, t_{0}\right)<4 \epsilon,
$$

which yields

$$
\hat{v}\left(p_{0}, t_{0}\right)<\psi\left(p_{0}, t_{0}\right)+4 \epsilon=\omega\left(p_{0}, t_{0}\right) .
$$

Thus, we have constructed $\omega \in \mathcal{S}$ that satisfies $\hat{v}\left(p_{0}, t_{0}\right)<\omega\left(p_{0}, t_{0}\right)$.

We then have the following existence theorem concerning parabolic viscosity solutions.

THEOREM 5.4. Let $f$ be a parabolic viscosity subsolution to (3.1), and $g$ be a parabolic viscosity supersolution to (3.1) satisfying $f \leq g$ on $\Omega_{T}$ and $f_{*}=g^{*}$ on $\partial_{\text {par }} \mathcal{O}_{0, T}$. Then there is a parabolic viscosity solution $u$ to (3.1) satisfying $u \in C\left(\overline{O_{T}}\right)$. Explicitly, there exists a unique parabolic viscosity infinite solution to Problem 4.1 when $h>1$.

\section{Proof. Let}

$S=\left\{v: v\right.$ is a parabolic viscosity subsolution to (3.1) in $\Omega_{T}$ with $v \leq g$ in $\left.\Omega_{T}\right\}$ and

$$
u(p, t)=\sup \{v(p, t): v \in S\} .
$$

Since $f \leq g$, the set $S$ is nonempty. Notice that $f \leq u \leq g$ by construction. By Lemma 5.2, $u$ is a parabolic viscosity subsolution. Suppose $u_{*}$ is not a parabolic viscosity supersolution. Then by Lemma 5.3 there exist a function $w \in S$ and 
a point $\left(p_{0}, t_{0}\right) \in \Omega_{T}$ such that $u\left(p_{0}, t_{0}\right)<w\left(p_{0}, t_{0}\right)$. But this contradicts the definition of $u$ at $\left(p_{0}, t_{0}\right)$. Thus, $u_{*}$ is a parabolic viscosity supersolution. By our assumptions on $f$ and $g$ on $\partial_{\text {par }} \mathcal{O}_{0, T}$,

$$
u=u^{*} \leq g^{*}=f_{*} \leq u_{*}
$$

on $\partial_{\text {par }} \mathcal{O}_{0, T}$. Then by the (assumed) comparison principle, $u \leq u_{*}$ on $\Omega_{T}$. Thus, we have that $u$ is a parabolic viscosity solution such that $u \in C\left(\overline{O_{T}}\right)$.

\subsection{The $h=1$ Case}

We begin by recalling the definition of upper and lower relaxed limits of a function $[8 ; 10]$.

Definition 5. For $\varepsilon>0$, consider the function $\mathfrak{h}_{\varepsilon}: O_{T} \subset G \rightarrow \mathbb{R}$. The upper relaxed limit $\overline{\mathfrak{h}}(p, t)$ and the lower relaxed limit $\underline{\mathfrak{h}}(p, t)$ are given by

$$
\begin{aligned}
\overline{\mathfrak{h}}(p, t) & =\limsup _{\hat{p} \rightarrow p, \hat{t} \rightarrow t, \varepsilon \rightarrow 0} \mathfrak{h}_{\varepsilon}(\hat{p}, \hat{t}) \\
& =\lim _{\varepsilon \rightarrow 0} \sup _{0<\delta<\varepsilon}\left\{\mathfrak{h}_{\delta}(\hat{p}, \hat{t}): O_{T} \cap B_{\varepsilon}(\hat{p}, \hat{t})\right\} \\
\text { and } \underline{\mathfrak{h}}(p, t) & =\liminf _{\hat{p} \rightarrow p, \hat{t} \rightarrow t, \varepsilon \rightarrow 0} \mathfrak{h}_{\varepsilon}(\hat{p}, \hat{t}) \\
& =\lim _{\varepsilon \rightarrow 0} \inf _{0<\delta<\varepsilon}\left\{\mathfrak{h}_{\delta}(\hat{p}, \hat{t}): O_{T} \cap B_{\varepsilon}(\hat{p}, \hat{t})\right\} .
\end{aligned}
$$

Taking the relaxed limits as $h \rightarrow 1^{+}$of the operator $F_{\infty}^{h}\left(\nabla_{0} u,\left(D^{2} u\right)^{\star}\right)$ in Equation (3.2), we have via the continuity of the operator

$$
\begin{aligned}
\bar{F}_{\infty}^{1}\left(\nabla_{0} u,\left(D^{2} u\right)^{\star}\right) & =\underline{F}_{\infty}^{1}\left(\nabla_{0} u,\left(D^{2} u\right)^{\star}\right) \\
& = \begin{cases}-\left\|\nabla_{0} u\right\|^{-2}\left\langle\left(D^{2} u\right)^{\star} \nabla_{0} u, \nabla_{0} u\right\rangle, & \nabla_{0} u \neq 0, \\
0, & \nabla_{0} u=0 .\end{cases}
\end{aligned}
$$

We give this operator the label $\mathcal{F}\left(\nabla_{0} u,\left(D^{2} u\right)^{\star}\right)$. Consider the relaxed limits $\bar{u}(p, t)$ and $\underline{u}(p, t)$ of the sequence of unique (continuous) viscosity solutions to Problem $4.1\left\{u_{h}(p, t)\right\}$ as $h \rightarrow 1^{+}$. By [10, Thm. 2.2.1] we have that $\bar{u}(p, t)$ is a viscosity subsolution and $\underline{u}(p, t)$ is a viscosity supersolution to

$$
u_{t}+\mathcal{F}\left(\nabla_{0} u,\left(D^{2} u\right)^{\star}\right)=0 .
$$

We have the following comparison principle, whose proof is similar to that of Theorem 4.3 in the case $h=1$ and is omitted.

Lemma 5.5. Let $\Omega$ be a bounded domain in $G$. If $\mathfrak{u}$ is a parabolic viscosity subsolution and $\mathfrak{v}$ a parabolic viscosity supersolution to

$$
u_{t}+\mathcal{F}\left(\nabla_{0} u,\left(D^{2} u\right)^{\star}\right)=0,
$$

then $\mathfrak{u} \leq \mathfrak{v}$ on $\Omega_{T} \equiv \Omega \times[0, T)$.

COROllary 5.6. $\bar{u}(p, t)=\underline{u}(p, t)$. 
Proof. By construction, $\underline{u}(p, t) \leq \bar{u}(p, t)$. By the lemma, $\underline{u}(p, t) \geq \bar{u}(p, t)$.

REMARK 5.7. Using the corollary, we will call this common relaxed limit $u^{1}(p, t)$. By [10, Chap. 2] and [8, Sect. 6], it is continuous, and the sequence $\left\{u_{h}(p, t)\right\}$ converges locally uniformly to $u^{1}(p, t)$ as $h \rightarrow 1^{+}$.

We then have the following theorem.

TheOREM 5.8. There exists a unique parabolic viscosity infinite solution to Problem 4.1 when $h=1$.

Proof. Let $\left\{u_{h}(p, t)\right\}$ and $u^{1}(p, t)$ be as before. Let $\left\{h_{j}\right\}$ be a subsequence with $h_{j} \rightarrow 1^{+}$where $u_{h}(p, t) \rightarrow u^{1}(p, t)$ uniformly. We may assume that $h_{j}<3$.

Let $\phi \in \mathcal{A} u_{1}\left(p_{0}, t_{0}\right)$. Using the uniform convergence, there is a sequence $\left\{p_{j}, t_{j}\right\} \rightarrow\left(p_{0}, t_{0}\right)$ such that $\phi \in \mathcal{A} u_{h_{j}}\left(p_{j}, t_{j}\right)$. If $\nabla_{0} \phi\left(p_{0}, t_{0}\right) \neq 0$, then we have $\nabla_{0} \phi\left(p_{j}, t_{j}\right) \neq 0$ for sufficiently large $j$. We then have

$$
\phi_{t}\left(p_{j}, t_{j}\right)-\Delta_{\infty}^{h_{j}} \phi\left(p_{j}, t_{j}\right) \leq 0
$$

and letting $j \rightarrow \infty$ yields

$$
\phi_{t}\left(p_{0}, t_{0}\right)-\Delta_{\infty}^{1} \phi\left(p_{0}, t_{0}\right) \leq 0
$$

Suppose $\nabla_{0} \phi\left(p_{0}, t_{0}\right)=0$. By Corollary 4.5 we may assume that $\left(D^{2} \phi\right)^{\star}\left(p_{0}\right.$, $\left.t_{0}\right)=0$. Passing to a subsequence if needed, we have $\nabla_{0} \phi\left(p_{j}, t_{j}\right) \neq 0$. Then

$$
\phi_{t}\left(p_{j}, t_{j}\right)-\max _{\|\eta\|=1}\left\langle\left(D^{2} \phi\right)^{\star}\left(p_{j}, t_{j}\right) \eta, \eta\right\rangle \leq \phi_{t}\left(p_{j}, t_{j}\right)-\Delta_{\infty}^{h_{j}} \phi\left(p_{j}, t_{j}\right) \leq 0 .
$$

Letting $j \rightarrow \infty$ yields

$$
\phi_{t}\left(p_{0}, t_{0}\right)=\phi_{t}\left(p_{j}, t_{j}\right)-\max _{\|\eta\|=1}\left\langle\left(D^{2} \phi\right)^{\star}\left(p_{0}, t_{0}\right) \eta, \eta\right\rangle \leq 0 .
$$

In the case $\nabla_{0} \phi\left(p_{j}, t_{j}\right)=0$, since $h_{j}<3$, we have $\phi_{t}\left(p_{j}, t_{j}\right) \leq 0$, and letting $j \rightarrow \infty$ yields $\phi_{t}\left(p_{0}, t_{0}\right) \leq 0$. We conclude that $u_{1}$ is a parabolic viscosity $h$ infinite subsolution. Similarly, $u_{1}$ is a parabolic viscosity $h$-infinite supersolution.

\section{The Limit as $t \rightarrow \infty$}

We now focus our attention on the asymptotic limits of the parabolic viscosity $h$-infinite solutions. We wish to show that for $1 \leq h$, we have that the (unique) viscosity solution to $u_{t}-\Delta_{\infty}^{h} u=0$ approaches the viscosity solution of $-\Delta_{\infty}^{h} u=$ 0 as $t \rightarrow \infty$. Our goal is the following theorem.

THEOREM 6.1. Let $h>1$, and let $u \in C(\bar{\Omega} \times[0, \infty))$ be a viscosity solution of

$$
\begin{cases}u_{t}-\Delta_{\infty}^{h} u=0 & \text { in } \Omega \times(0, \infty) \\ u(p, t)=g(p) & \text { on } \partial_{\mathrm{par}}(\Omega \times(0, \infty))\end{cases}
$$

with $g: \bar{\Omega} \rightarrow \mathbb{R}$ continuous and assuming that $\partial \Omega$ satisfies the property of positive geometric density (see $[11, p$. 2,909]). Then $u(p, t) \rightarrow U(p)$ uniformly in $\Omega$ 
as $t \rightarrow \infty$ where $U(p)$ is the unique viscosity solution of $-\Delta_{\infty}^{h} U=0$ with the Dirichlet boundary condition $\lim _{q \rightarrow p} U(q)=g(p)$ for all $p \in \partial \Omega$.

We first must establish the uniqueness of viscosity solutions to the limit equation. Note that for future reference, we include the case $h=1$.

Theorem 6.2. Let $1 \leq h<\infty$, and let $\Omega$ be a bounded domain. Let $u$ be a viscosity subsolution to $\Delta_{\infty}^{h} u=0$, and let $v$ be a viscosity supersolution to $-\Delta_{\infty}^{h} u=0$. Then,

$$
\sup _{p \in \bar{\Omega}}(u(p)-v(p))=\sup _{p \in \partial \Omega}(u(p)-v(p)) .
$$

Proof. Let $u$ be a viscosity subsolution to $-\Delta_{\infty}^{h} u=0$. Then choose $\phi \in \mathcal{C}_{\text {sub }}^{2}(\Omega)$ such that $0=\phi\left(p_{0}\right)-u\left(p_{0}\right)<\phi(p)-u(p)$ for $p \in \Omega, p \neq p_{0}$. If $\left\|\nabla_{0} \phi\left(p_{0}\right)\right\|=$ 0 , then $-\left\langle\left(D^{2} \phi\right)^{\star}\left(p_{0}\right) \nabla_{0} \phi\left(p_{0}\right), \nabla_{0} \phi\left(p_{0}\right)\right\rangle=0 \leq 0$. If $\left\|\nabla_{0} \phi\left(p_{0}\right)\right\| \neq 0$, we then have

$$
-\Delta_{\infty}^{h} \phi\left(p_{0}\right)=-\left\|\nabla_{0} \phi\left(p_{0}\right)\right\|^{h-3}\left\langle\left(D^{2} \phi\right)^{\star}\left(p_{0}\right) \nabla_{0} \phi\left(p_{0}\right), \nabla_{0} \phi\left(p_{0}\right)\right\rangle \leq 0 .
$$

Dividing, we have $-\left\langle\left(D^{2} \phi\right)^{\star}\left(p_{0}\right) \nabla_{0} \phi\left(p_{0}\right), \nabla_{0} \phi\left(p_{0}\right)\right\rangle \leq 0$. In either case, $u$ is a viscosity subsolution to $-\Delta_{\infty}^{3} u=0$. Similarly, $v$ is a viscosity supersolution to $-\Delta_{\infty}^{3} u=0$. The theorem follows from the corresponding result for $-\Delta_{\infty}^{3} u=0$ in $[5 ; 3 ; 14]$.

We state some obvious corollaries.

Corollary 6.3. Let $1 \leq h<\infty$, and let $g: \partial \Omega \rightarrow \mathbb{R}$ be continuous. Then there is exactly one solution to

$$
\begin{cases}-\Delta_{\infty}^{h} u=0 & \text { in } \Omega, \\ u=g & \text { on } \partial \Omega .\end{cases}
$$

Corollary 6.4. Let $1 \leq h<\infty$, and let $g: \partial \Omega \rightarrow \mathbb{R}$ be continuous. The unique viscosity solution to

$$
\begin{cases}-\Delta_{\infty}^{h} u=0 & \text { in } \Omega, \\ u=g & \text { on } \partial \Omega\end{cases}
$$

is the unique viscosity solution to

$$
\begin{cases}-\Delta_{\infty}^{3} u=0 & \text { in } \Omega, \\ u=g & \text { on } \partial \Omega .\end{cases}
$$

Our method of proof for Theorem 6.1 follows that of [11, Thm. 2], the core of which hinges on the construction of a parabolic test function from an elliptic one. In order to construct such a parabolic test function, we need to examine the homogeneity of Equation (6.1). A quick calculation shows that for a fixed $h>1$, $k^{1 /(h-1)} u(x, k t)$ is a $\mathcal{C}_{\text {sub }}^{2}$ solution to Equation (6.1) if $u(x, t)$ is a $\mathcal{C}_{\text {sub }}^{2}$ solution. A routine calculation then shows that parabolic viscosity $h$-infinite solutions share 
this homogeneity. We use this property in the following lemma, the proof of which can be found in [9, p. 170]. (Also, cf. [6, Lemma 6.2] and [11].)

Lemma 6.5. Let $u$ be as in Theorem 6.1, and $h>1$. Then for every $(x, t) \in \Omega \times$ $(0, \infty)$ and for $0<\mathcal{T}<t$, we have

$$
|u(x, t-\mathcal{T})-u(x, t)| \leq \frac{2\|g\|_{\infty, \Omega}}{h-1}\left(1-\frac{\mathcal{T}}{t}\right)^{h /(1-h)} \frac{\mathcal{T}}{t} .
$$

Proof of Theorem 6.1. Fix $h>1$. Let $u$ be a viscosity solution of (6.1). The results of [9, Chap. III] imply that the family $\{u(\cdot, t): t \in(0, \infty)\}$ is equicontinuous. Since it is uniformly bounded due to the boundedness of $g$, ArzelàAscoli's theorem yields that there exists a sequence $t_{j} \rightarrow \infty$ such that $u\left(\cdot, t_{j}\right)$ converge uniformly in $\bar{\Omega}$ to a function $U \in C(\bar{\Omega})$ for which $U(p)=g(p)$ for all $p \in \partial \Omega$. By Corollary 6.3 it suffices to show that $U$ is a viscosity subsolution to $-\Delta_{\infty}^{h} U=0$ on $\Omega$. With that in mind, let $p_{0} \in \Omega$ and choose $\phi \in \mathcal{C}_{\text {sub }}^{2}(\Omega)$ such that $0=\phi\left(p_{0}\right)-U\left(p_{0}\right)<\phi(p)-U(p)$ for $p \in \Omega, p \neq p_{0}$. Using the uniform convergence, we can find a sequence $p_{j} \rightarrow p_{0}$ such that $u\left(\cdot, t_{j}\right)-\phi$ has a local maximum at $p_{j}$. Now define

$$
\phi_{j}(p, t)=\phi(p)+C\left(\frac{t}{t_{j}}\right)^{h /(1-h)} \frac{t_{j}-t}{t_{j}},
$$

where $C=2\|g\|_{\infty, \Omega} /(h-1)$. Note that $\phi_{j}(p, t) \in \mathcal{C}_{\text {sub }}^{2}(\Omega \times(0, \infty))$. Then using Lemma 6.5, we have

$$
\begin{aligned}
u\left(p_{j}, t_{j}\right)-\phi_{j}\left(p_{j}, t_{j}\right) & =u\left(p_{j}, t_{j}\right)-\phi\left(p_{j}\right) \geq u\left(p, t_{j}\right)-\phi(p) \\
& \geq u(p, t)-\phi(p)-C\left(\frac{t}{t_{j}}\right)^{h /(1-h)} \frac{t_{j}-t}{t_{j}} \\
& =u(p, t)-\phi_{j}(p, t)
\end{aligned}
$$

for any $p \in \Omega$ and $0<t<t_{j}$. Thus, we have that $\phi_{j}$ is an admissible test function at $\left(p_{j}, t_{j}\right)$ on $\Omega \times[0, T]$. Therefore,

$$
\left(\phi_{j}\right)_{t}\left(p_{j}, t_{j}\right)-\Delta_{\infty}^{h} \phi_{j}\left(p_{j}, t_{j}\right) \leq 0 .
$$

This yields

$$
-\Delta_{\infty}^{h} \phi\left(p_{j}\right) \leq \frac{C}{t_{j}}
$$

The theorem follows by letting $j \rightarrow \infty$.

Combining the results of the previous sections, we have the following theorem.

THEOREM 6.6. Let $\Omega$ be a bounded domain where $\partial \Omega$ satisfies the property of positive geometric density. Let $h \geq 1$, and let $g: \bar{\Omega} \rightarrow \mathbb{R}$ be continuous. Let $u^{h, t}$ be the unique viscosity solution to

$$
\begin{cases}u_{t}^{h, t}-\Delta_{\infty}^{h} u^{h, t}=0 & \text { in } \Omega \times(0, \infty) \\ u^{h, t}(p, t)=g(p) & \text { on } \partial_{\mathrm{par}}(\Omega \times(0, \infty))\end{cases}
$$


and let $u^{h, \infty}$ be the unique viscosity solution to

$$
\begin{cases}-\Delta_{\infty}^{h} u^{h, \infty}=0 & \text { in } \Omega \\ u^{h, \infty}=g & \text { on } \partial \Omega\end{cases}
$$

with the Dirichlet boundary condition $\lim _{q \rightarrow p} u^{h, \infty}(q)=g(p)$ for all $p \in \partial \Omega$. Then

$$
\lim _{\substack{h \rightarrow 1^{+} \\ t \rightarrow \infty}} u^{h, t}=\lim _{h \rightarrow 1^{+}} \lim _{t \rightarrow \infty} u^{h, t}=\lim _{t \rightarrow \infty} \lim _{h \rightarrow 1^{+}} u^{h, t}=u^{1, \infty} .
$$

That is, the following diagram commutes:

$$
\begin{aligned}
& u_{t}^{h, t}-\Delta_{\infty}^{h} u^{h, t}=0 \underset{h \rightarrow 1^{+}}{\longrightarrow} u_{t}^{1, t}-\Delta_{\infty}^{1} u^{1, t}=0 \\
& \downarrow t \rightarrow \infty \quad \downarrow t \rightarrow \infty \\
& -\Delta_{\infty}^{h} u^{h, \infty}=0 \underset{h \rightarrow 1^{+}}{\longrightarrow}-\Delta_{\infty}^{1} u^{1, \infty}=0
\end{aligned}
$$

Proof. By Theorem 6.1,

$$
\lim _{t \rightarrow \infty} u^{h, t}=u^{h, \infty},
$$

and the convergence is uniform. By Corollary 6.4,

$$
\lim _{h \rightarrow 1^{+}} u^{h, \infty}=u^{1, \infty},
$$

and this convergence is clearly uniform. We thus have the iterated limit

$$
\lim _{h \rightarrow 1^{+}} \lim _{t \rightarrow \infty} u^{h, t}=u^{1, \infty}
$$

with both limits converging uniformly. By Remark 5.7 we have

$$
\lim _{h \rightarrow 1^{+}} u^{h, t}=u^{1, t},
$$

and this convergence is locally uniform. By the proof of Theorem 6.1 we have

$$
\lim _{t \rightarrow \infty} u^{1, t}=f
$$

for some function $f$, and the convergence is uniform. We then have

$$
\lim _{t \rightarrow \infty} \lim _{h \rightarrow 1^{+}} u^{h, t}=f
$$

with the inner limit locally uniform and the outer limit uniform. By the results of iterated limits in [1, Sect. 19] we then have that the full (double) limit exists. In addition, the full limit and both iterated limits equal. That is, $f=u^{1, \infty}$ and

$$
\lim _{\substack{h \rightarrow 1^{+} \\ t \rightarrow \infty}} u^{h, t}=\lim _{h \rightarrow 1^{+}} \lim _{t \rightarrow \infty} u^{h, t}=\lim _{t \rightarrow \infty} \lim _{h \rightarrow 1^{+}} u^{h, t}=u^{1, \infty} .
$$




\section{References}

[1] R. Bartle, The elements of real analysis, 2nd edition, John Wiley and Sons, New York, 1976.

[2] A. Bellaïche, The tangent space in sub-Riemannian geometry, Sub-Riemannian geometry (A. Bellaïche, J.-J. Risler, eds.), Progr. Math., 144, pp. 1-78, Birkhäuser, Basel, Switzerland, 1996.

[3] T. Bieske, On infinite harmonic functions on the Heisenberg group, Comm. Partial Differential Equations 27 (2002), no. 3\&4, 727-762.

[4] Comparison principle for parabolic equations in the Heisenberg group, Electron. J. Differ. Equ. 2005 (2005), no. 95, 1-11.

[5] _ A sub-Riemannian maximum principle and its application to the $p$ Laplacian in Carnot groups, Ann. Acad. Sci. Fenn. Math. 37 (2012), 119-134.

[6] T. Bieske and E. Martin, The parabolic p-Laplace equation in Carnot groups, Ann. Acad. Sci. Fenn. Math. 39 (2014), 605-623.

[7] N. Bourbaki, Lie groups and Lie algebras, Chapters 1-3, Elements of Mathematics, Springer-Verlag, Berlin, 1989.

[8] M. Crandall, H. Ishii, and P.-L. Lions, User's guide to viscosity solutions of second order partial differential equations, Bull. Amer. Math. Soc. 27 (1992), no. 1, 1-67.

[9] E. DiBenedetto, Degenerate parabolic equations, Springer-Verlag, New York, 1993.

[10] Y. Giga, Surface evolution equations: a level set approach, Monogr. Math., 99, Birkhäuser Verlag, Basel, Switzerland, 2006.

[11] P. Juutinen, On the definition of viscosity solutions for parabolic equations, Proc. Amer. Math. Soc. 129 (2001), no. 10, 2907-2911.

[12] P. Juutinen and B. Kawohl, On the evolution governed by the infinite Laplacian, Math. Ann. 335 (2006), no. 4, 819-851.

[13] M. Portilheiro, Vázquez, and J. Luis, Degenerate homogeneous parabolic equations associated with the infinity-Laplacian, Calc. Var. Partial Differential Equations 46 (2013), no. 3-4, 705-724.

[14] C. Y. Wang, The Aronsson equation for gradient minimizers of $L^{\infty}$-functionals associated with vector fields satisfying Hörmander's condition, Trans. Amer. Math. Soc. 359 (2007), 91-113.

T. Bieske

Department of Mathematics and Statistics

University of South Florida

4202 E. Fowler Ave. CMC342

Tampa, FL 33620

USA

tbieske@usf.edu
E. Martin

Department of Mathematics and Physics

Westminster College

501 Westminster Ave

Fulton, MO 65251

USA 